\title{
BMJ Open Can Schwartz Center Rounds support healthcare staff with emotional challenges at work, and how do they compare with other interventions aimed at providing similar support? A systematic review and scoping reviews
}

Cath Taylor, ${ }^{1,2}$ Andreas Xyrichis, ${ }^{2}$ Mary C Leamy, ${ }^{2}$ Ellie Reynolds, ${ }^{2}$ Jill Maben ${ }^{1,2}$

To cite: Taylor C, Xyrichis A, Leamy MC, et al. Can Schwartz Center Rounds support healthcare staff with emotional challenges at work, and how do they compare with other interventions aimed at providing similar support? A systematic review and scoping reviews. BMJ Open 2018;8:e024254. doi:10.1136/ bmjopen-2018-024254

- Prepublication history and additional material for this paper are available online. To view these files, please visit the journal online (http://dx.doi org/10.1136/bmjopen-2018024254).

Received 17 May 2018

Revised 27 July 2018

Accepted 23 August 2018
Check for updates

(C) Author(s) (or their employer(s)) 2018. Re-use permitted under CC BY. Published by BMJ.

${ }^{1}$ School of Health Sciences, University of Surrey, Guildford, UK

${ }^{2}$ Florence Nightingale Faculty of Nursing, Midwifery \& Palliative Care, King's College London, London, UK

Correspondence to

Dr. Cath Taylor;

cath.taylor@surrey.ac.uk

\section{ABSTRACT}

Objectives (i) To synthesise the evidence-base for Schwartz Center Rounds (Rounds) to assess any impact on healthcare staff and identify key features; (ii) to scope evidence for interventions with similar aims, and compare effectiveness and key features to Rounds.

Design Systematic review of Rounds literature; scoping reviews of comparator interventions (action learning sets; after action reviews; Balint groups; caregiver support programme; clinical supervision; critical incident stress debriefing; mindfulness-based stress reduction; peersupported storytelling; psychosocial intervention training; reflective practice groups; resilience training).

Data sources PsychINFO, CINAHL, MEDLINE and EMBASE, internet search engines; consultation with experts.

Eligibility criteria Empirical evaluations (qualitative or quantitative); any healthcare staff in any healthcare setting; published in English.

Results The overall evidence base for Rounds is limited. We developed a composite definition to aid comparison with other interventions from 41 documents containing a definition of Rounds. Twelve (10 studies) were empirical evaluations. All were of low/moderate quality (weak study designs including lack of control groups). Findings showed the value of Rounds to attenders, with a self-reported positive impact on individuals, their relationships with colleagues and patients and wider cultural changes. The evidence for the comparative interventions was scant and also low/moderate quality. Some features of Rounds were shared by other interventions, but Rounds offer unique features including being open to all staff and having no expectation for verbal contribution by attenders.

Conclusions Evidence of effectiveness for all interventions considered here remains limited. Methods that enable identification of core features related to effectiveness are needed to optimise benefit for individual staff members and organisations as a whole. A systems approach conceptualising workplace well-being arising from both individual and environmental/structural factors, and comprising interventions both for assessing and improving the well-being of healthcare staff, is required.
Strengths and limitations of this study

- This is the first systematic review of Schwartz Center Rounds (Rounds), a healthcare staff intervention from the USA that has spread rapidly through UK healthcare organisations.

- Additional scoping reviews of 11 interventions with similar aims to support the well-being of healthcare staff, enables a novel comparative analysis to key features of Schwartz Rounds.

- This paper compares other staff well-being interventions to Rounds, thereby resulting in a focus on key features of Rounds; we did not explicitly draw out key features of other interventions or compare them against each other.

- The use of scoping reviews for comparator interventions, and exclusion of evidence in populations other than healthcare staff means that some evidence may have been omitted.

- The heterogeneity of study designs and outcomes, and weak study designs, means that findings are summarised narratively rather than using meta-analysis.

Schwartz Rounds could be considered as one strategy to enhance staff well-being.

\section{INTRODUCTION}

In this paper, we report the systematic review of evidence regarding Schwartz Center Rounds (Rounds) and conduct a comparative analysis of 11 interventions also broadly aimed at supporting healthcare staff with the emotional challenges of their work. In doing so, we define Rounds from the literature and discuss the future potential use of interventions to support staff with the emotional challenges of providing healthcare. Healthcare providers are among the largest employers in 
many countries worldwide. For example, the UK National Health Service (NHS) employs 1.5 million staff, ${ }^{1}$ and in 2014 there were approximately 1.8 million physicians, ${ }^{2}$ and 3.4 million nurses ${ }^{3}$ across the European Union. Provision of healthcare relies on both clinical and non-clinical staff (eg, managers, administrators, porters/orderlies, caterers and domestic staff), all of whom may be impacted by the emotional challenges they face in their interactions with the patients and families they come across in day-to-day life.

Numerous publications have highlighted the high prevalence of psychological morbidity among healthcare staff in both clinical and non-clinical roles, and in many different countries worldwide. ${ }^{4-10}$ Indeed, studies have typically reported between a quarter to a third of healthcare staff to have levels of psychological distress indicative of the need for clinical intervention, and in the UK mental health reasons explain a third of all NHS sickness absence, costing approximately $£ 1$ billion (of the total $£ 2.4$ billion cost of sickness absence in 2015). ${ }^{11}$ Together with the clear consequences of this for their well-being and quality of life, and impact on their families, there is now increasing recognition of the link between the well-being of healthcare staff and quality of patient care (in relation to both patient experience and clinical outcomes). ${ }^{12-15}$

Consequently, the well-being of healthcare staff is high on the agenda of healthcare organisations in the UK and worldwide ${ }^{16-21}$ In the UK, the National Institute for Health and Care Excellence guidance published in 2009 recommended that organisations take a strategic approach to tackling staff well-being, encompassing approaches that focus on both prevention and treatment and that include interventions for individuals as well as 'organisation-wide approaches that encompass all employees'. ${ }^{22}$ However, the reviews underpinning this guidance highlighted the poor quality of evidence overall and in particular the limited evidence on organisation-wide policies or approaches, with the strongest evidence in relation to interventions aimed at stress management for individuals. ${ }^{22}$

Schwartz Rounds are a rare example of an organisation-wide intervention that has seen rapid spread across healthcare organisations in the UK. ${ }^{23}$ Rounds originated in the USA where they now run in over 430 organisations. After a pilot introduction to two UK hospitals in 2009, they now run in over 170 UK health and social care organisations (hospitals, hospices, community settings). They were developed to support healthcare staff to deliver compassionate care by providing a safe space where staff could openly share and reflect on the emotional, social and ethical challenges faced at work. The premise is that caregivers will be more able to make personal connections with colleagues and patients if they have insight into their own responses and feelings. Their rapid adoption in the UK was despite a limited evidence base, although attendance at Rounds was reported to be associated with improved compassion for patients, better teamwork and reduced stress in staff members, as well as having a positive impact on organisational culture. ${ }^{24} 25$
Consequently, the National Institute for Health Research commissioned a national evaluation of Rounds that has recently concluded ${ }^{26}$ supporting these earlier findings and showing attendance at Rounds to be associated with a reduction in psychiatric morbidity. A key component of the evaluation, intended to support organisational decision-making regarding staff well-being interventions, was to review the evidence for Schwartz Rounds and contextualise them by comparing the features of Rounds to other staff well-being interventions with similar aims. This paper reports the results from this, and thereby aims to answer the following review questions:

1. What are the defining features of Schwartz Center Rounds, and what is their evidence base?

2. What comparable interventions providing staff support/reflective space exist, what key features do they share with Schwartz Rounds and what is their evidence base in healthcare professionals?

Specifically, we aim to (i) identify key features of Rounds by synthesising published descriptions of Rounds to create a composite definition; (ii) systematically review and appraise all empirical evaluations of Rounds; (iii) identify comparative interventions, describe their key features and scope their evidence base and (iv) document similarities and differences between Rounds and comparative interventions.

\section{METHODS}

The review of Schwartz Rounds literature followed Preferred Reporting Items for Systematic Reviews and Meta-Analyses systematic literature review guidance where applicable.

\section{Search strategy}

The search strategies for the systematic review of Rounds literature involved: (i) a traditional database search (PsychINFO, CINAHL, MEDLINE and EMBASE to give comprehensive coverage of medical, psychological, nursing and social sciences literature). As an example, the MEDLINE database search for Schwartz Rounds was: (Schwartz adj2 Round*).mp. ( $\mathrm{mp}=$ title, abstract, original title, name of substance word, subject heading word, keyword heading word, protocol supplementary concept word, rare disease supplementary concept word, unique identifier), (ii) use of internet search engines and (iii) consultation with experts. Inclusion criteria included having a health professional sample (either qualified or trainee) and empirically evaluating the intervention using qualitative and/or quantitative methods. The review excluded non-English language sources, unpublished dissertations/theses and any papers not accessible via the institution's online library, Google Scholar or directly from the journal website. All records were pooled together into a bibliographic database. First, records were screened to exclude duplicate entries. Second, the title and abstract of remaining records was reviewed for eligibility. All database searches were conducted between 14 
October 2014 and 5 February 2015, although searches for Schwartz Rounds evaluations and consultation with experts continued until September 2017.

\section{Data extraction and quality appraisal}

Standard data items were extracted to describe included papers (eg, citation, country, setting, population/sample, overall design, etc) and the evaluation (eg, length of evaluation; data collection method/s; outcome measures; key findings) using extraction sheets that were developed and piloted by all data extractors. In addition, items were developed that were specific to each intervention, for example, whether group or individual focused, size of group, length/number of sessions, content of sessions, whether facilitated or not (and if facilitated whether training/supervision was provided). Quality assessment of qualitative and quantitative primary studies was undertaken for each study using the tools developed by Jones $e t$ $a l,{ }^{27}$ which include assessment of key criteria and then an overall rating (high—no or few flaws; moderate-some flaws; low-significant flaws). Mixed methods studies were, in addition, assessed against the six criteria for good reporting of mixed methods studies developed by O'Cathain et al. ${ }^{28}$ Quality was rated low ( $<3$ criteria were met); moderate (3-4) or high (5+).

\section{Synthesis}

Thematic analysis of the types of outcomes reported resulted in the identification of three categories relating to: a) self; b) others (eg, patients, colleagues) or c) wider organisation (eg, changes to policies; organisational metrics such as safety or satisfaction). Findings are presented according to these three categories. Finally, the overall quality of the evidence base for each intervention is described based on the range in quality for individual studies.

\section{CONSTRUCTING A COMPOSITE DEFINITION OF SCHWARTZ ROUNDS}

While the Schwartz Center for Compassionate Care, where Rounds originated, have a description of Schwartz Rounds on their website, this was found to omit key aspects of their design that we knew from scoping the literature to be important (eg, an ongoing programme, time-fixed in length, food is provided, it is open to all staff and panellists stories are preprepared). Therefore, we constructed a 'composite' definition based on descriptions used in Rounds literature in order to determine the key features of Rounds for comparison with other interventions. For this process, we included all literature (including non-empirical literature, eg, letters, editorials) providing it included a description of Rounds. Text describing Rounds (what they were and their intended aims, eg, structure and purpose, as well as any text describing what they were 'not') was extracted from published accounts. The text was analysed thematically by four team members independently (CT, JM, ML, MH), core concepts were discussed and agreed and a single definition was produced. The face validity of the definition was confirmed after review by study advisory and steering group members.

\section{SCOPING REVIEWS OF COMPARATIVE INTERVENTIONS}

The reviews of comparable interventions followed an interpretative scoping literature review methodology based on the framework outlined by Arksey and O'Malley. ${ }^{29}$ The searching, data extraction and synthesis followed similar steps to the review of Schwartz Rounds literature (except where noted below) but instead of producing a detailed critique and review of individual studies they were instead aimed at producing a summary description of the evidence base in relation to size, scope and quality, and used to extract data relevant for the comparative analysis. For each intervention, the number/ type of included papers was recorded, and each intervention was described in relation to its original format (eg, number of participants, original setting and healthcare setting/s and intended aims/outcomes); and the variability in its application within the literature (fidelity to original format). Main findings were examined across all interventions and analysed thematically (using the same categories as for Schwartz Rounds: self, others, organisation) to enable synthesis within, and comparison across, each intervention.

\section{Identification of comparative interventions to include}

We aimed to identify interventions that support health professionals with the emotional challenges of delivering patient care. Initially, we identified aspects that were fundamental to Rounds, including providing an opportunity for reflection, disclosure and offering psychological safety; and these informed choices regarding potential comparative interventions. Included interventions needed to focus on psychological (as opposed to physical) well-being of staff; be person-directed (vs work directed) and provide primarily emotional rather than cognitive/ clinical support (eg, excluding mortality/morbidity meetings, which aim to provide lessons in terms of cognitive errors or systems issues). Although Rounds are a 'group' (rather than individual) intervention, we chose not to limit comparative interventions by this characteristic, due to the importance of reflection and/or disclosure as a key potential mechanism in Rounds that is shared by other interventions that are not group-based. Potential comparative interventions were identified through published reviews of psychological/emotional support interventions for healthcare staff ${ }^{30-33}$ and through consultation with steering and advisory group members (with expertise in Rounds/well-being interventions in healthcare). A total of 11 interventions were scoped: action learning sets; after action reviews; Balint groups; caregiver support programme; clinical supervision; critical incident stress debriefing; mindfulness-based stress reduction; 
peer-supported storytelling; psychosocial intervention training; reflective practice groups; resilience training.

\section{Comparative analysis to Schwartz Rounds}

The composite definition of Rounds was disaggregated into its individual descriptive features which were extracted into a table, together with the features that were 'not' part of Rounds. Further clarification was added for some descriptive features to ensure clarity of meaning (eg, 'reflection' became 'provides an explicit opportunity for reflection'). The description of each comparative intervention was then reviewed by the research team and assessed in relation to whether or not it also provided each of the key features of Rounds. The face validity of the comparison between Rounds and other interventions was confirmed with study advisory and steering groups (with expertise in Rounds/healthcare staff well-being interventions).

\section{PATIENT INVOLVEMENT}

We actively involved patients through membership of the Project Steering Group (PSG), which included two patient public involvement (PPI) representatives (Havi Carel, Christine Chapman) who had previously provided input to the original funding application. The PSG provided oversight to all aspects of the study, and alongside other group members our PPI representatives and Rounds staff members advised on design, inclusion of comparative interventions and commented on the findings.

\section{RESULTS}

\section{Key features of Rounds}

Forty-three documents/sources were included in the review of descriptions of Rounds (table 1), which allowed development of the definition. ${ }^{24} 25$ 34-74 The majority $(n=33)$ were non-empirical publications (eg, commentaries, descriptive reports of a single Round). The thematic synthesis resulted in the production of the composite definition (see online supplementary file 1), a summary version is provided in table 1 .

\section{EVIDENCE BASE FOR ROUNDS: RESULTS FROM THE SYSTEMATIC REVIEW}

Twelve empirical evaluations of Rounds were included (table 2) arising from 10 studies (4 in the USA, 6 in the UK). Most were mixed methods evaluations, typically comprising attenders completing evaluation forms postRound attendance, followed by interviews or focus groups $(n=5)$, one mixed method study comprised case studies (observation/interviews) together with descriptive analysis of evaluation forms ${ }^{75}$ and one used both quantitative and qualitative methods to analyse evaluation forms. ${ }^{76}$ Two were quantitative studies, and one qualitative study. Only one study included non-attenders ${ }^{66}$ (table 2).
Overall quality of the evidence-base was assessed to be low/moderate. Most studies had study designs prone to risk of bias (eg, cross-sectional), used non-validated questionnaires (typically self-report views/ satisfaction with Rounds and impact of attendance) and none of the quantitative evaluations had control group (non-attender) comparisons. Little information was provided on the samples/sampling frames in quantitative studies (eg, in relation to breadth of professional group representation or role in Rounds), nor were findings analysed or presented in relation to such factors. In two studies that did report the characteristics of their quantitative sample, most were female and of white ethnicity, and nurses predominated (but neither study reported the seniority of nurse). ${ }^{24} 47$ Findings from these studies included that Rounds are highly valued by attenders (although represented a small proportion of total staff). Most studies reported positive impact on 'self' (eg, improved well-being, coping) 2425444749515966707576 and impact on patients (increased compassion, empathy) 2425515966707576 and colleagues (improved teamwork, compassion/empathy)..$^{24254447505159667576} \mathrm{Six}$ studies provide evidence of wider institutional impacts from interviews with attenders ${ }^{24} 254451667576$ (table 2). Three of the included studies were evaluations of Rounds adapted for educational purposes ${ }^{39} 5070$; all reporting that Rounds were felt to be useful and that students gained knowledge/understanding about the emotional side of providing patient care.

\section{COMPARATIVE INTERVENTIONS: RESULTS FROM THE SCOPING REVIEWS}

Electronic searches for the 11 comparative interventions yielded a total of 1725 papers, of which 146 were included (ranging between 1 and 64 across interventions, table 1 , see online supplementary file 2 for included references). A number of publications $(n=253)$ were not obtainable due to being published in sources that no longer existed or not available through institutional subscription and internet searches. The largest evidence base was for clinical supervision $(n=64)$ followed by Balint groups $(n=26)$. Half of the studies were quantitative $(n=74$ : RCT, observational, quasi-experimental), 41 were qualitative (mixed designs, interviews, focus groups), 22 were mixed methods and 9 were secondary studies (literature reviews). The literature was international with the majority of studies from the USA and the UK; other countries represented included Canada, Australia, Finland, Norway, Sweden, Croatia, Spain, Italy, Israel and South Africa. There was a distinct lack of studies from Asia, although that may be a reflection of the English language limit.

For most interventions, high-quality evidence was sparse. Populations for many of the interventions lacked diversity across health professions and settings, with many mostly nursing-focused. The aims of studies varied widely, with a few aimed at assessing efficacy or effectiveness but most were 


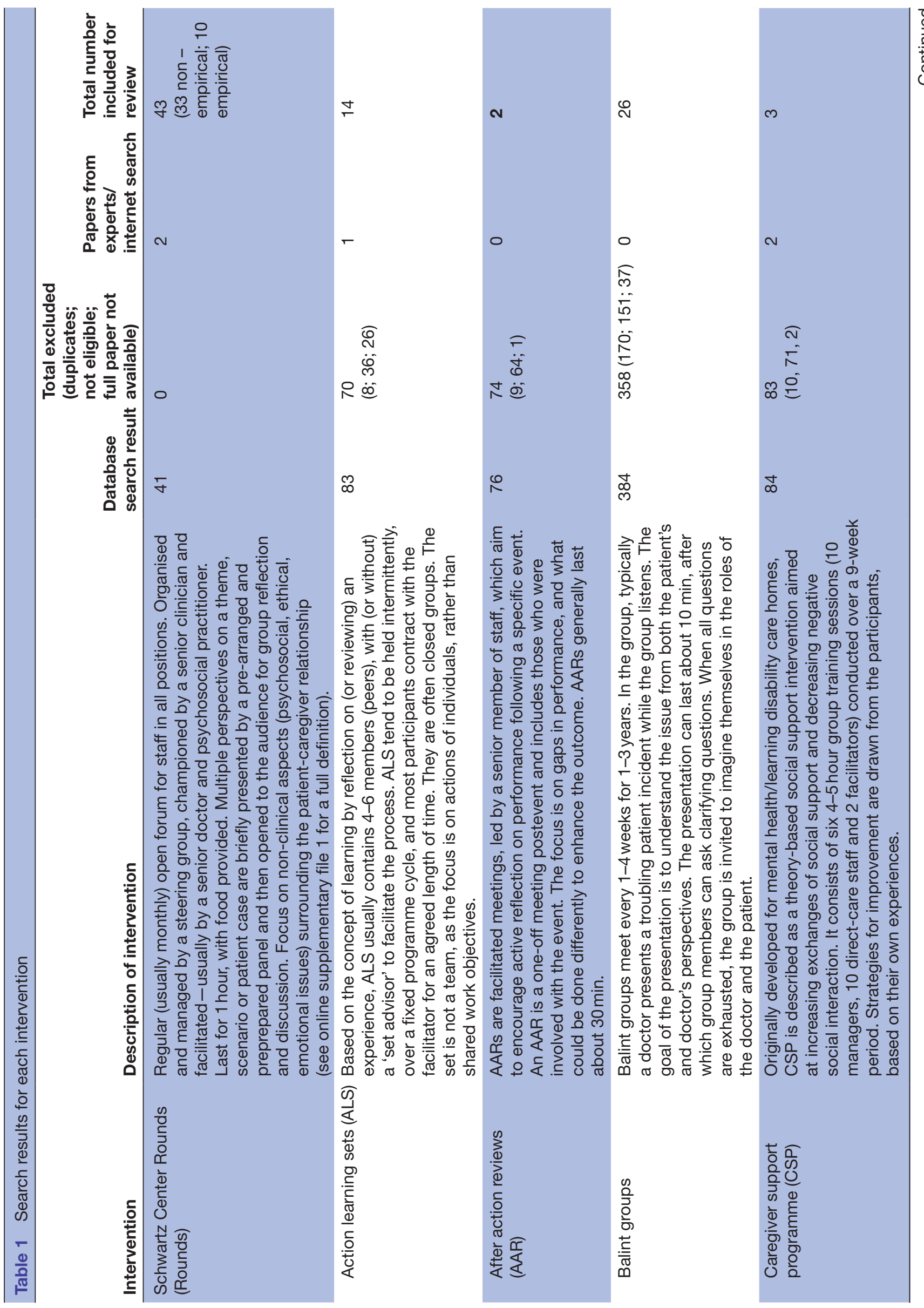




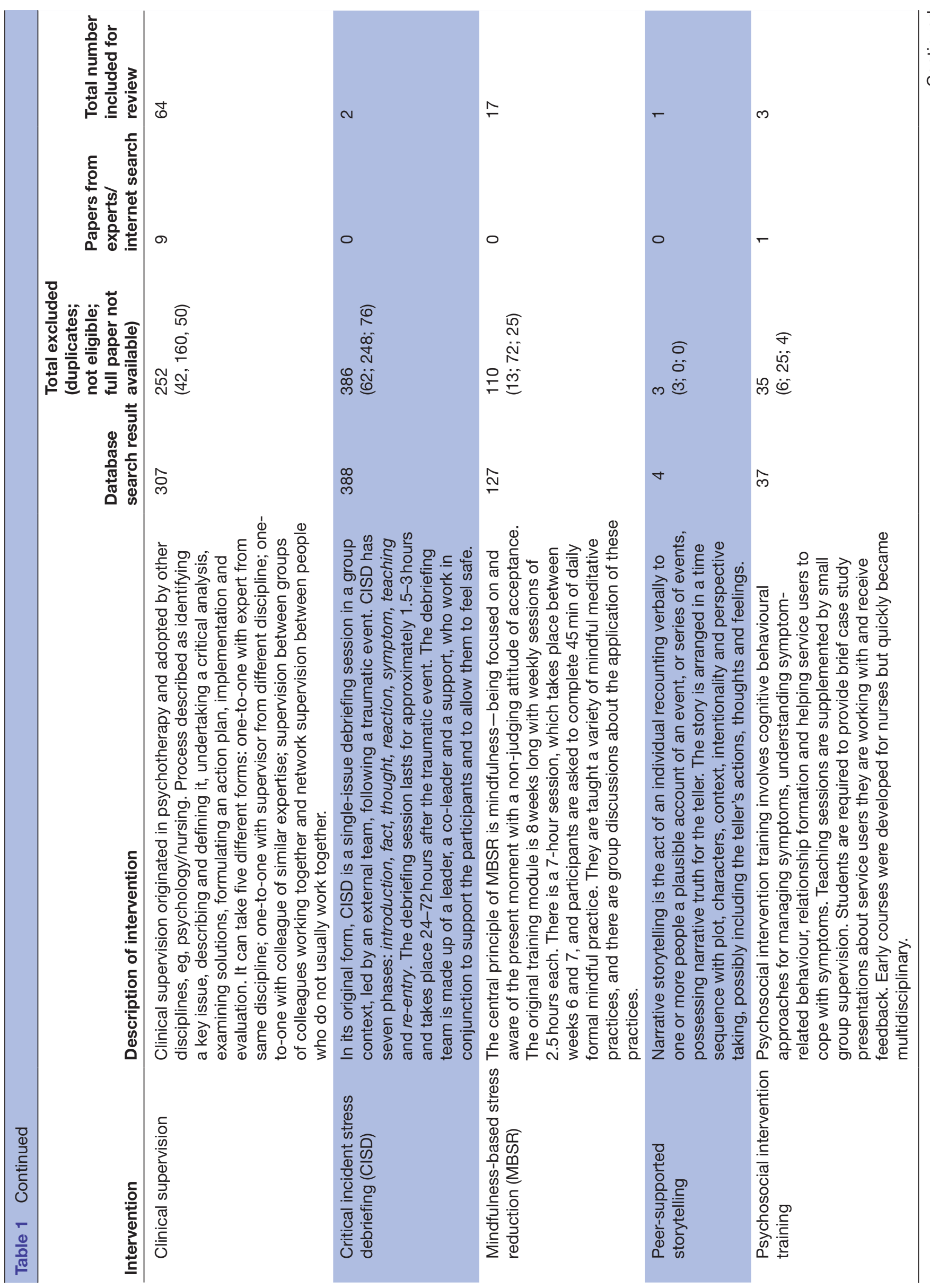

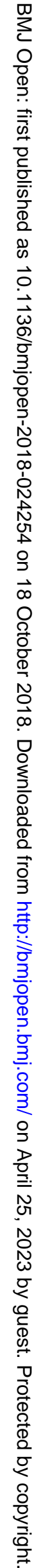




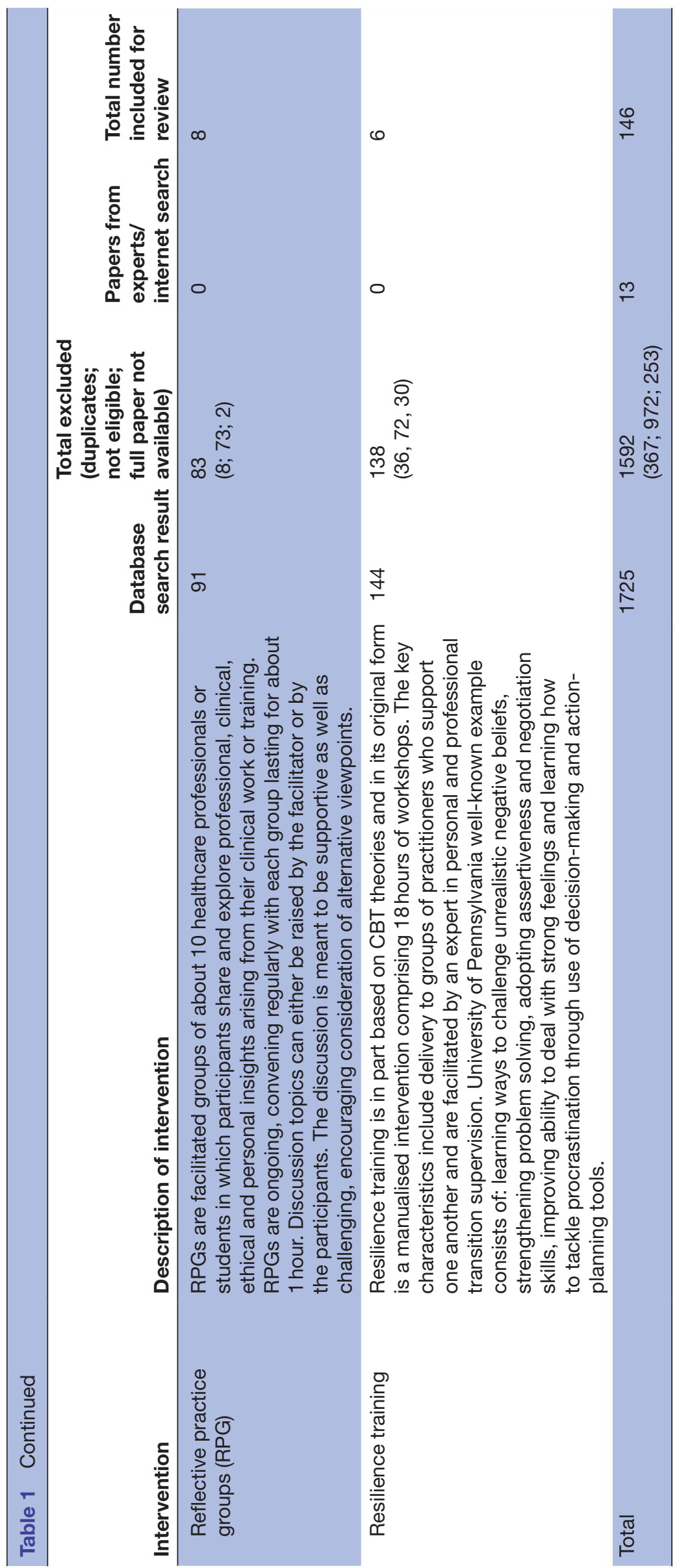




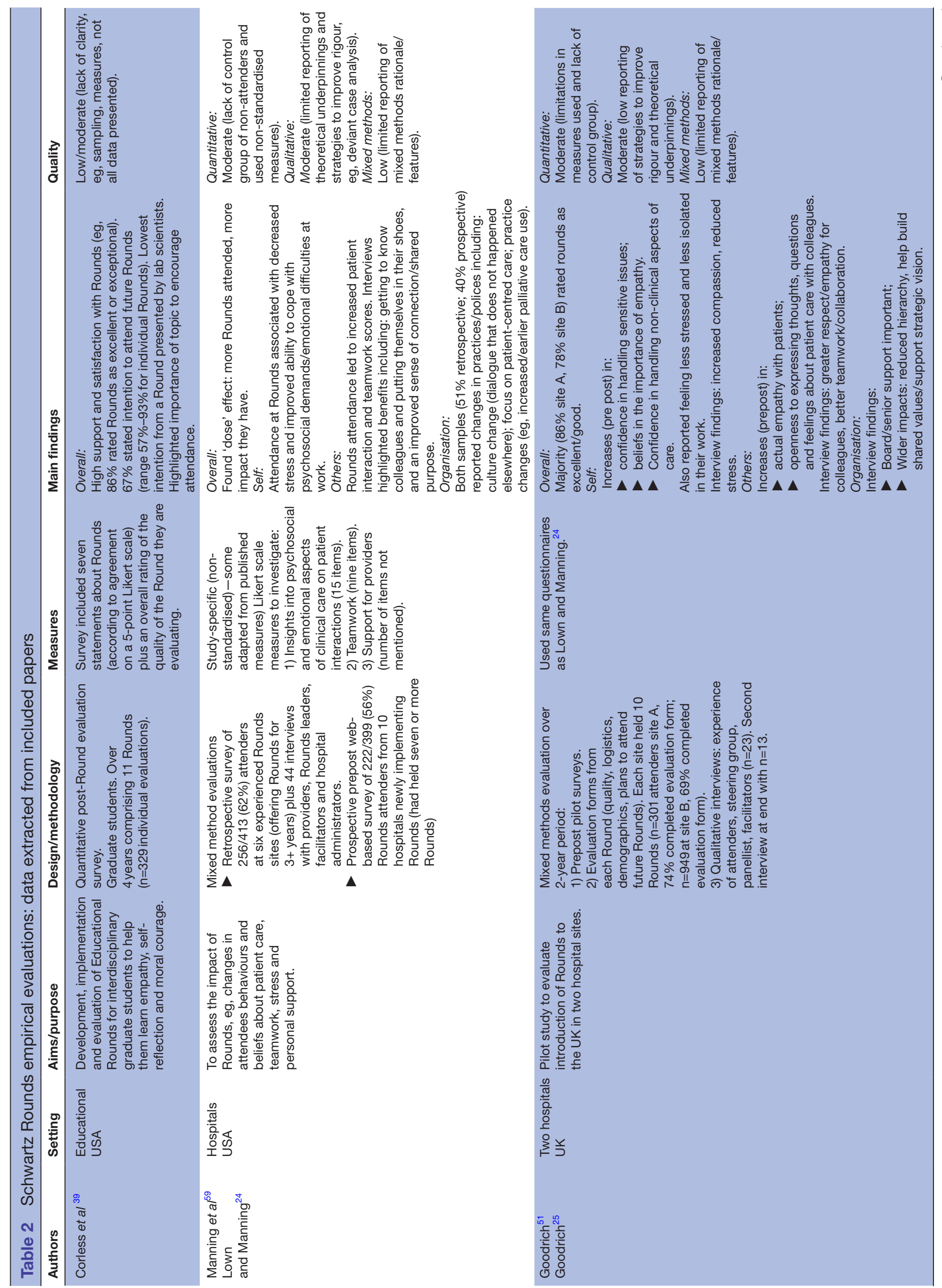




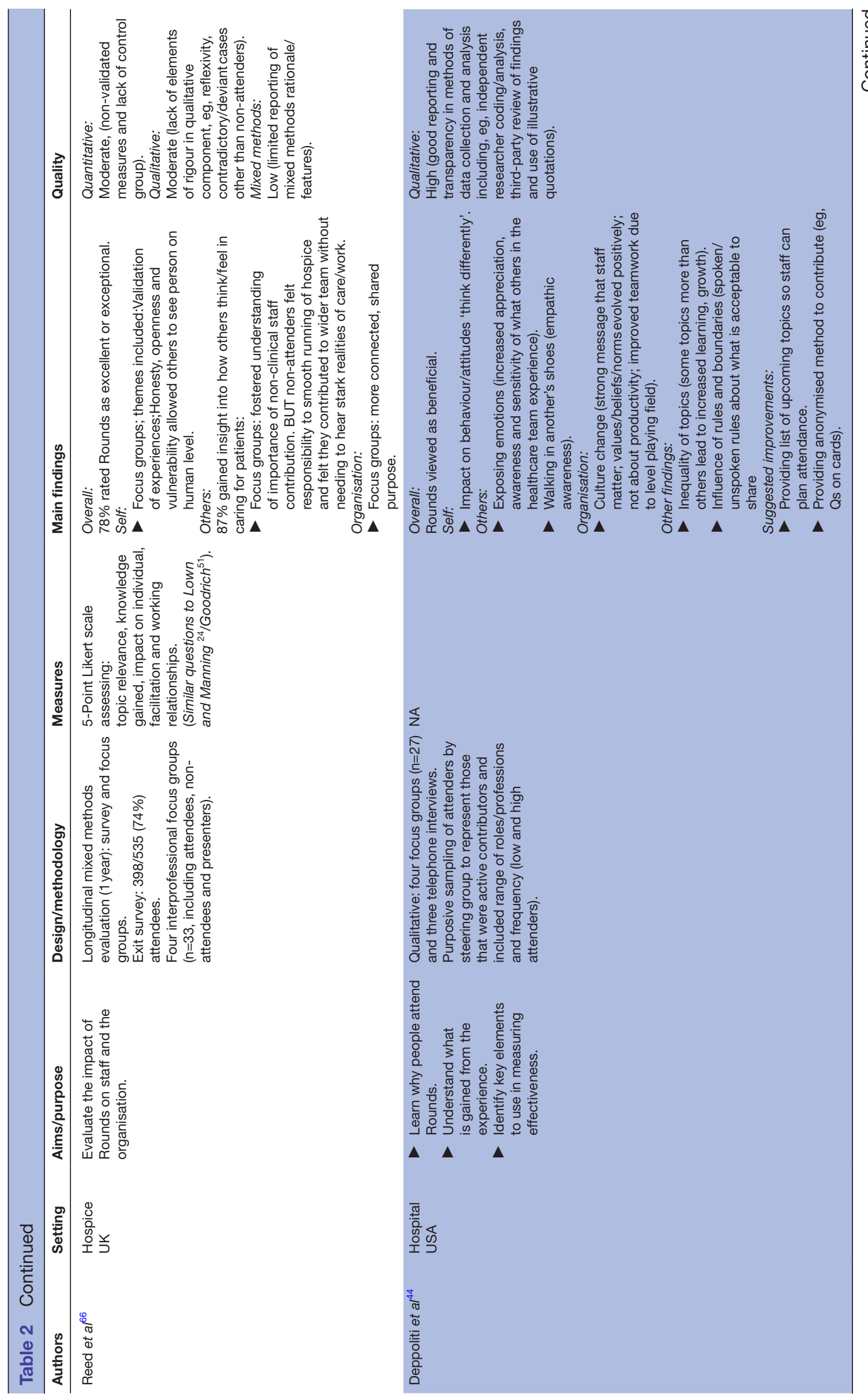




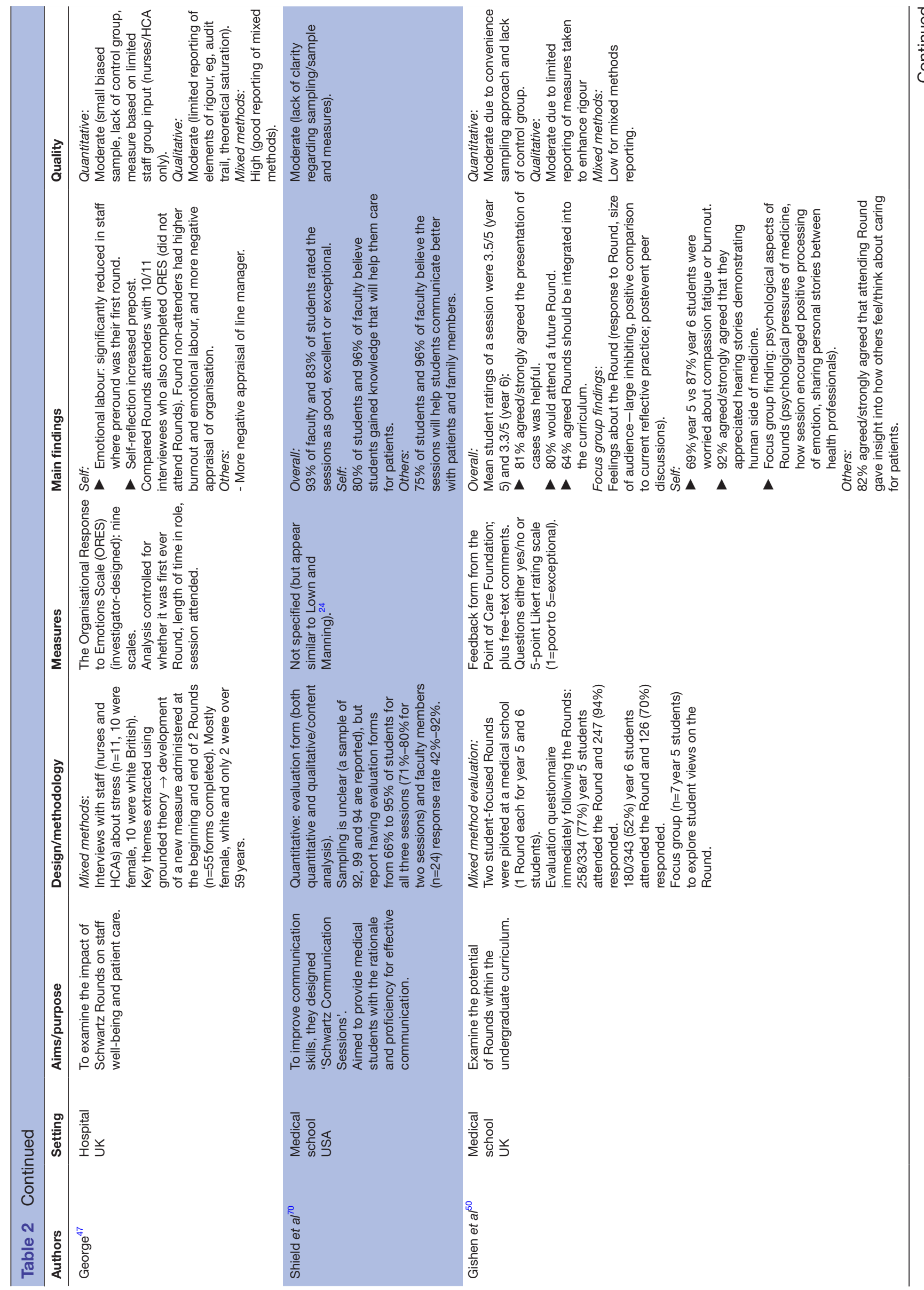




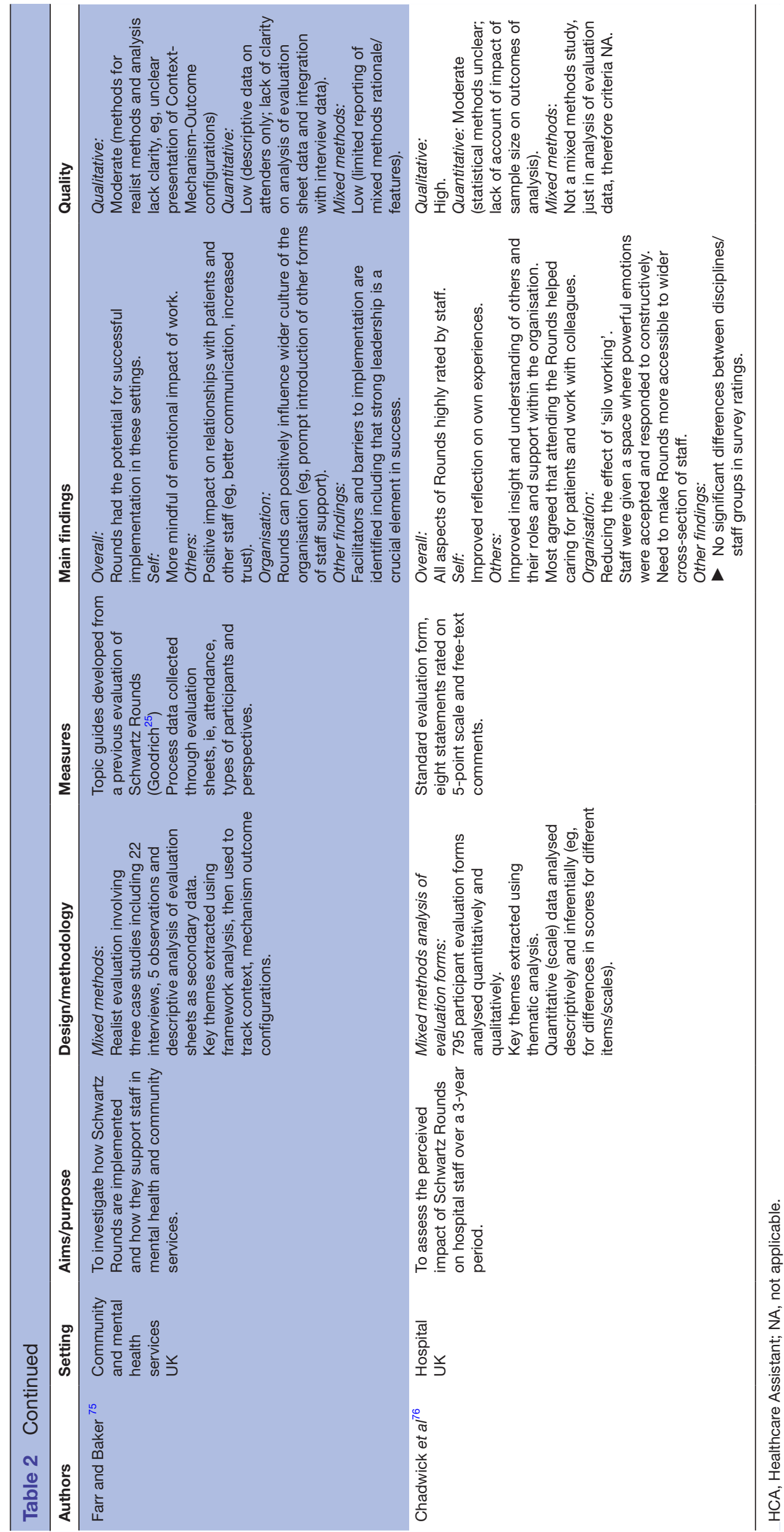


small-scale exploratory descriptive studies. The content and format of interventions (fidelity) was in most cases widely heterogeneous (and/or lacked detail), and consequently synthesis of findings is problematic. Most of the quantitative evaluations across all interventions relied on weaker study designs (eg, cross-sectional studies, postintervention evaluations, lacking control comparisons), used non-probability sampling, had small samples likely to be underpowered and used non-validated outcomes measures. Many qualitative studies also lacked clear reporting of aspects of rigour (eg, limited reporting of member checking, deviant cases, reflexivity or evidence of data saturation). A summary of the evidence base for each intervention is provided in online supplementary file 3 .

\section{Synthesis}

Most interventions presented evidence in relation to all three categories of outcomes ('self', 'others' and 'organisation'), although evidence for resilience training, mindfulness-based stress reduction and reflective practice groups lacked inclusion of organisational outcomes. All of the interventions had evidence of positive benefits to self (eg, raised self-awareness, resilience, job satisfaction, empowerment or overall well-being), and most provided some evidence of positive benefits to 'others'. Impact on patients included fostering of better provider-service user relationships, communication with and/or attitudes towards patients and improved patient-centredness, knowledge of patients' suffering and empathy. Impact on colleagues, included associations with better teamwork, peer support and knowledge/understanding of colleagues.

At organisational level, there was evidence from some interventions of association with improved practice, for example, reductions in unnecessary prescriptions, increased uptake of psychosocial support (Balint groups), reduction in task and coordination errors and increased uptake of postfall huddles (after action reviews). Two interventions provided evidence of a positive impact on the workforce, including providing opportunities for mentoring and advice (action learning sets) and improved staff retention (clinical supervision).

\section{SCHWARTZ VERSUS ALTERNATIVE INTERVENTIONS: COMPARATIVE FEATURES}

In comparison to the other interventions, Rounds offer a unique organisation-wide 'all-staff' forum to share stories about the emotional impact of providing patient care (table 3). While many of the other interventions expect 'open, honest communication' as a key feature, and provide an explicit opportunity for reflection, none is open to all staff (eg, clinical and non-clinical, voluntary attendance) and many are not ongoing programmes but instead are one-off training courses or events. Some of the training interventions (eg, mindfulness-based stress reduction, or resilience training) are multidisciplinary in training attendance, but conduct/practice of the intervention occurs subsequently and is individual, compared with Schwartz Rounds (and other interventions such as Balint groups), where learning and practice take place simultaneously in group settings.

Other key aspects in which Rounds are distinct from the comparative interventions relate to what Rounds are intentionally 'not' meant to be. In particular, discussions within Rounds should not 'problem solve' in order to avoid focus on the clinical decision-making in a patient case, whereas problem solving/action planning are key features of many of the other interventions (eg, action learning sets, after action reviews, critical incident stress debriefing). Most of the comparative interventions also offered flexibility in format, compared with Rounds which require a contractual licence (with stipulated conditions) obtained via the Schwartz Center for Compassionate Care (USA) or Point of Care Foundation (PoCF, UK).

Arguably the closest types of interventions to Rounds are Balint groups (although rooted in unidisciplinary primary care-physicians only-with closed membership), and reflective practice groups (again generally closed membership and can be unidisciplinary). In particular, both are ongoing group programmes in which challenging/rewarding experiences about delivering patient care are shared and discussions are facilitated, and both provide the opportunity to give and/or receive peer support in safe and confidential environments. However, neither offers an organisation-wide opportunity for staff to attend, and both would have an expectation that members/attenders would contribute, whereas in Rounds attenders can choose to be silent listeners. Clinical supervision can also provide an opportunity to reflect on the emotional and ethical challenges of care without problem-solving/action planning,but unlike Rounds this usually occurs in a one-to-one situation, not group, and requires those being supervised to verbally contribute.

\section{DISCUSSION}

Our work revealed a rich portfolio of available interventions to support staff with the emotional challenges of providing healthcare, each designed with different audiences and uses in mind. The evidence base regarding the effectiveness of these largely remains weak, and more should be done to examine these more systematically. The studies reviewed here show some evidence of impact at different levels, and future work should seek to unpick which interventions work best, under which conditions and for which participants. To our knowledge, this is the first comparative review of staff well-being interventions.

Given the high rates of work-related stress and mental health issues among healthcare staff, it is not acceptable for employers not to act, despite the weak evidence base for most approaches and interventions currently. Some staff groups have clinical supervision, for example, as an integral part of their work (mental health nurses; midwives; psychologists and social workers), whereas most doctors and nurses do not, and such staff often have little or no support with the emotional, social and 
Table 3 Features of Rounds compared/contrasted with comparative interventions

\begin{tabular}{|c|c|c|c|c|c|c|c|c|c|c|c|c|}
\hline & \multirow[b]{2}{*}{ Feature of Rounds } & \multicolumn{11}{|c|}{ Intervention (see footnote for full label) } \\
\hline & & ALS & AAR & Balint & Care & Super & CISD & Mind & Story & Psych & Refl & Resil \\
\hline 1 & $\begin{array}{l}\text { Share challenging/rewarding } \\
\text { experiences about delivering } \\
\text { patient care }\end{array}$ & $\begin{array}{l}\text { May } \\
\text { Not }\end{array}$ & No & Yes & $\begin{array}{l}\text { May } \\
\text { Not }\end{array}$ & Yes & Yes & No & $\begin{array}{l}\text { May } \\
\text { Not }\end{array}$ & Yes & Yes & $\begin{array}{l}\text { May } \\
\text { Not }\end{array}$ \\
\hline 2 & $\begin{array}{l}\text { Focus on psychosocial and } \\
\text { emotional issues of patient- } \\
\text { caregiver relationships }\end{array}$ & $\begin{array}{l}\text { May } \\
\text { Not }\end{array}$ & No & Yes & $\begin{array}{l}\text { May } \\
\text { Not }\end{array}$ & May Not & $\begin{array}{l}\text { May } \\
\text { Not }\end{array}$ & No & $\begin{array}{l}\text { May } \\
\text { Not }\end{array}$ & May Not & $\begin{array}{l}\text { May } \\
\text { Not }\end{array}$ & $\begin{array}{l}\text { May } \\
\text { Not }\end{array}$ \\
\hline 3 & $\begin{array}{l}\text { Provides an explicit opportunity } \\
\text { for reflection }\end{array}$ & Yes & Yes & Yes & $\begin{array}{l}\text { May } \\
\text { Not }\end{array}$ & Yes & Yes & Yes & Yes & Yes & Yes & $\begin{array}{l}\text { May } \\
\text { Not }\end{array}$ \\
\hline 4 & Open, honest communication & Yes & Yes & Yes & Yes & Yes & Yes & Yes & Yes & Yes & Yes & $\begin{array}{l}\text { May } \\
\text { Not }\end{array}$ \\
\hline 5 & $\begin{array}{l}\text { Provides an opportunity to give } \\
\text { and/or receive peer support }\end{array}$ & Yes & $\begin{array}{l}\text { May } \\
\text { Not }\end{array}$ & Yes & Yes & $\begin{array}{l}\text { Yes (if } \\
\text { group) }\end{array}$ & Yes & Yes & Yes & No & Yes & Yes \\
\hline 6 & $\begin{array}{l}\text { Telling and hearing stories } \\
\text { related to a theme, scenario or } \\
\text { patient case }\end{array}$ & No & Yes & No & $\begin{array}{l}\text { May } \\
\text { Not }\end{array}$ & No & No & No & Yes & No & No & No \\
\hline 7 & $\begin{array}{l}\text { Ongoing programme (vs one- } \\
\text { off) }\end{array}$ & No & No & Yes & No & Yes & No & No & No & No & Yes & No \\
\hline 8 & $\begin{array}{l}\text { Time-fixed session (vs flexible } \\
\text { length/unspecified) }\end{array}$ & No & No & Yes & Yes & Yes & No & Yes & No & Yes & No & Yes \\
\hline 9 & $\begin{array}{l}\text { Planned provision of food/ } \\
\text { refreshments }\end{array}$ & No & No & No & No & No & No & No & No & No & No & No \\
\hline 10 & $\begin{array}{l}\text { Open to all/any clinical and } \\
\text { non-clinical staff }\end{array}$ & No & Yes & No & No & No & No & No & No & No & No & $\begin{array}{l}\text { May } \\
\text { Not }\end{array}$ \\
\hline 11 & $\begin{array}{l}\text { All levels of staff/intended to } \\
\text { flatten hierarchy }\end{array}$ & Yes & Yes & No & Yes & No & No & Yes & Yes & Yes & No & Yes \\
\hline 12 & $\begin{array}{l}\text { Open group membership (vs } \\
\text { closed/invited members only) }\end{array}$ & No & No & No & No & No & No & No & No & No & No & No \\
\hline 13 & Multidisciplinary & $\begin{array}{l}\text { May } \\
\text { Not }\end{array}$ & Yes & $\begin{array}{l}\text { May } \\
\text { Not }\end{array}$ & Yes & No & $\begin{array}{l}\text { May } \\
\text { Not }\end{array}$ & Yes & $\begin{array}{l}\text { May } \\
\text { Not }\end{array}$ & Yes & No & $\begin{array}{l}\text { May } \\
\text { Not }\end{array}$ \\
\hline 14 & $\begin{array}{l}\text { Preprepared/rehearsed stories } \\
\text { or focus }\end{array}$ & Yes & No & No & No & No & No & No & Yes & No & No & No \\
\hline 15 & Facilitated discussions & $\begin{array}{l}\text { May } \\
\text { Not }\end{array}$ & Yes & Yes & Yes & Yes & Yes & Yes & No & Yes & Yes & Yes \\
\hline 16 & $\begin{array}{l}\text { Panel presenters tell stories } \\
\text { giving their perspectives on a } \\
\text { theme, scenario or patient case }\end{array}$ & No & No & No & No & No & No & No & No & No & No & No \\
\hline 17 & Group intervention & Yes & Yes & Yes & Yes & May Not & Yes & Yes & No & Yes & Yes & Yes \\
\hline 18 & $\begin{array}{l}\text { Organisational support: senior } \\
\text { doctor/clinician champions }\end{array}$ & $\begin{array}{l}\text { May } \\
\text { Not }\end{array}$ & Yes & Yes & Yes & Yes & $\begin{array}{l}\text { May } \\
\text { Not }\end{array}$ & No & No & No & No & No \\
\hline 19 & $\begin{array}{l}\text { Safe and confidential } \\
\text { environment }\end{array}$ & Yes & Yes & Yes & Yes & Yes & Yes & Yes & Yes & Yes & Yes & Yes \\
\hline
\end{tabular}

Feature of Rounds

Intervention (see footnote for full label)

\begin{tabular}{lllllllllllll}
\hline \multirow{2}{*}{$\begin{array}{l}\text { Features that define what Rounds } \\
\text { are 'not' }\end{array}$} & Intervention & ALS & AAR & Balint & Care & Super & CISD & Mind & Story & Psych & Refl & Resil \\
\hline 1 & Problem-solving & Yes & Yes & Yes & Yes & $\begin{array}{l}\text { May } \\
\text { Not }\end{array}$ & No & No & Yes & No & Yes \\
\hline 2 & $\begin{array}{l}\text { Production of actionable } \\
\text { outputs }\end{array}$ & Yes & Yes & No & No & May Not No & No & No & No & No & No \\
3 & $\begin{array}{l}\text { Flexibility in format (vs licensed/ Yes } \\
\text { contract* }^{*}\end{array}$ & Yes & Yes & $\begin{array}{l}\text { May } \\
\text { Not }\end{array}$ & Yes & Yes & Yes & Yes & No & Yes & May \\
Not
\end{tabular}


Table 3 Continued

\begin{tabular}{llllllllllll} 
Features that define what Rounds & Intervention \\
\cline { 2 - 9 } are 'not' & ALS & AAR & Balint & Care & Super & CISD & Mind & Story & Psych & Refl & Resil \\
\hline 4 & $\begin{array}{l}\text { Focus on clinical aspects of } \\
\text { patient care, their diagnosis or }\end{array}$ & May & May & No & May & May Not May & No & May & Yes & May & May \\
& Not & & Not & & Not & & Not & & Not & Not
\end{tabular}

plan of care

*Licensed/contract-fidelity to original intervention, ie, one model/approaches or many, degree of flexibility offered.

AAR, after action reviews; ALS, action learning sets; Balint, Balint groups; Care, caregiver support programme; CISD, critical incident stress debriefing; Mind, mindfulness-based stress reduction; Psych, psychosocial intervention training; Refl, reflective practice groups; Resil, resiliencetraining; Story, peer-supported story-telling; Super, clinical/restorative supervision.

ethical challenges of their work. Non-clinical staff-who may have much contact with patients and the events they encounter-are even more neglected in relation to the impact of delivering patient care on them. Selection of interventions should be based on a strategic approach that incorporates needs assessment, implementation of interventions/approaches and policies and monitoring and review to determine the impact of these and refine/revise as necessary. There is a need for a range of approaches, not a one-size-fits-all and our work does not suggest an either/or approach for individual interventions. Rounds should not be seen as a replacement for or instead of clinical supervision (or other support/interventions), but could be offered to staff in addition. Organisation-wide interventions are important to tackle workplace environmental/cultural factors that impact on well-being; to change attitudes and cultural norms around staff needing support as well as changing conversations in organisations around empathy, compassion and the support required to deliver these. Involving all employees may improve coworker and supervisor support, which in turn can facilitate the development of a supportive workplace environment that reduces stress by improving attitudes and behaviours. ${ }^{77}$ Compared with other interventions reviewed here, Rounds offer a unique organisation-wide 'all staff' forum to reflect on the emotional impact of providing patient care, offering opportunities for staff to reflect, whether or not they choose to disclose/contribute to discussions, and accruing evidence suggests they may have many benefits to individuals, others (colleagues, patients) as well as wider organisational impacts. ${ }^{26}$

Schwartz Rounds were originally conceived to meet a very specific identified need in healthcare: to support healthcare providers to be compassionate to patients through giving them insight into their own thoughts, feelings and behaviours. ${ }^{26}$ In the UK, the reasons given for adoption has been more about staff well-being, in line with evidence linking quality of patient care and experience with staff well-being. ${ }^{13}{ }^{23}$ Unlike many of the other interventions, they have a structured format, and are specifically not intended to be 'problem-solving'. In doing so, they provide a 'counter-cultural' space that differs from the protocol-driven, outcome-orientated healthcare environment that values emotional stoicism: 'Good Rounds shift an organisation and its workers away from their default position of urgent action, reaction and problem solving to an hour of stillness and slowness ${ }^{78}$ (p. 41). A key ingredient supporting Rounds to meet their intended aims is good facilitation, thus the role of the facilitator is key. Unlike the facilitation role in other interventions we reviewed, where there was often much variability in relation 'fidelity', in the UK, it is mandatory for Rounds facilitators to attend training provided by the PoCF (the UK licence holder for Schwartz Rounds), and they receive ongoing support from Schwartz mentors. It is recommended that there are at least two facilitators in each organisation, and the PoCF state that it helps if facilitators have experience of group work, and managing difficult emotions (many have psychology or social work backgrounds). In our national evaluation, we found despite most having these skills and background, they often shouldered the responsibility for Rounds on their own (some having only one facilitator too), which we found to impact negatively on their well-being, and on the sustainability of Rounds, recommending that a focus on facilitator support, and succession planning would be beneficial for Rounds ${ }^{2679}$

Workforce interventions are often complex in nature, with many components and aims. Their evaluation is thereby challenging, particularly with regard to attributing any changes to outcomes to the intervention as opposed to other causes within the organisation/system. The challenge of conducting a robust evaluations of organisation-wide interventions may be one explanation as to why such evidence is so sparse, ${ }^{22}$ and for why there is instead a predominance of evidence regarding individually targeted interventions such as mindfulness-based stress reduction. The application of new methodologies to address these challenges, such as realist evaluation, could enable a more robust understanding of how and why interventions work (or do not work), and has recently been applied in the first UK national evaluation of Schwartz Rounds. ${ }^{26}$

\section{Limitations}

The focus of this review on the evidence within healthcare staff meant that wider evidence for some interventions, beyond healthcare, was not considered. Also, the scoping methods applied to the comparable interventions inevitably means that some relevant evidence may have been omitted, although systematic electronic searching and consultation with experts aimed to minimise this risk. The rapid uptake of Rounds in the UK and need to contextualise them within 
staff well-being interventions, informed the design of this review. It was thereby a review that compared other interventions with the key features of Rounds, and did not thereby compare the key features of all the other interventions with each other, apart from by describing and synthesising their origins and evidence base.

\section{CONCLUSION}

Given the time and resources already committed to the interventions considered here, it is important to determine how best to identify the core features of effectiveness to optimise benefit for individual staff members and organisations as a whole. This work has now been undertaken for Schwartz Rounds using a realist-informed methodology that has identified the contextual factors that influence how and for whom Schwartz Rounds work, resulting in an organisational guide giving practical guidance and recommendations for organisations to maximise the effectiveness of Rounds in their organisations. ${ }^{2679}$ The application of similar methodologies for other interventions such as clinical supervision and Balint groups may further help ensure optimal outcomes. A systems approach as opposed to an individual approach to tackling staff well-being, in order to improve patient care, is required, comprising effective interventions for assessing and improving the well-being of healthcare staff .

Acknowledgements The authors would like to thank the members of the project advisory group (Joanna Goodrich, Barbara Wren, Jenny Firth-Cozens, Michael West, John Pepper, Glenn Robert) and steering group (Dean Fathers, Sharon Fleming, Nicola Mackintosh, Rachel Massey-Chase, Katherine Hopkins, Jude Bayly, Havi Carel, Christine Chapman, Elisabeth Buggins, Jennifer Gardner, Ruth Harris and Lisl Klein) for their guidance and comments throughout the study. The authors would also like to thank Michelle Hope for her contribution to the analysis of definitions of Schwartz Rounds.

Contributors CT and JM conceived the original idea and designed the study; AX designed the search strategies and performed the database searches; CT, AX, ML and ER performed internet searches; screened titles and abstracts independently; extracted data and assessed quality for three interventions each; CT, JM, MCL and ER created the composite definition of Rounds. All authors contributed to the overall synthesis and comparative analysis; CT drafted the manuscript; AX, MCL, ER and $\mathrm{JM}$ revised critically the manuscript. All the authors approved the final version of the manuscript.

Funding This work was supported by the National Institute for Health Research Health Services and Delivery Research Programme (project number 13/07/49).

Disclaimer The views and opinions expressed therein are those of the authors and do not necessarily reflect those of the NHS, the NIHR, MRC, CCF, NETSCC, the HS\&DR programme or the Department of Health.

Competing interests $\mathrm{JM}$ reports that she was a member of an advisory group from 2006 to 2009, advising on the development of the Point of Care project at The King's Fund, and a member of the Point of Care Foundation Board 2013-2014; she stepped down as boardmember at the start of the evaluation. All other authors declare no conflicts of interest.

Patient consent Not required.

Provenance and peer review Not commissioned; externally peer reviewed.

Data sharing statement № additional data are available.

Open access This is an open access article distributed in accordance with the Creative Commons Attribution 4.0 Unported (CC BY 4.0) license, which permits others to copy, redistribute, remix, transform and build upon this work for any purpose, provided the original work is properly cited, a link to the licence is given, and indication of whether changes were made. See: https://creativecommons.org/ licenses/by/4.0/.

\section{REFERENCES}

1. NHS (2018) https://www.nhs.uk/NHSEngland/thenhs/about/Pages/ overview.aspx. (Last accessed 15 Feb 18)

2. Europa Eurostat (2018) http://ec.europa.eu/eurostat/statisticsexplained/index.php/Healthcare_personnel_statistics_-_physicians. (Last accessed 15 Feb 18).

3. Europa Eurostat (2018) http://ec.europa.eu/eurostat/statisticsexplained/index.php/Healthcare_personnel_statistics_-_nursing_ and_caring_professionals (Last accessed 15 Feb 18).

4. Taylor C, Graham J, Potts H, et al. Changes in mental health of UK hospital consultants since the mid-1990s Lancet. , 2005:366, 742-4.

5. Wall TD, Bolden RI, Borrill CS, et al. Minor psychiatric disorder in NHS trust staff: occupational and gender differences. Br J Psychiatry 1997;171:519-23.

6. Maslach C, Jackson SE. Burnout in health professionals: a social psychological analysis. In: Sanders GS, Suls J, eds. Social Psychology of Health and Illness. Hillsdale NJ: Lawrence Erlbaum Associates, 1982.

7. Arigoni F, Bovier PA, Mermillod B, et al. Prevalence of burnout among Swiss cancer clinicians, paediatricians and general practitioners: who are most at risk? Support Care Cancer 2009;17:75-81.

8. Trufelli DC, Bensi CG, Garcia JB, et al. Burnout in cancer professionals: a systematic review and meta-analysis. Eur $\mathrm{J}$ Cancer Care 2008:17:31.

9. Bressi C, Manenti S, Porcellana M, et al. Haemato-oncology and burnout: an Italian survey. Br J Cancer 2008;98:1046-52.

10. Harvey SB, Laird B, Henderson M, et al. The mental health of health care professionals: a review for the Department of Health. Department of Health, London 2009.

11. Quality Watch (2018). http://www.qualitywatch.org.uk/indicator/nhsstaff-sickness-absence\# (Last accessed 15 Feb 18).

12. Taylor $\mathrm{C}$, Graham J, Potts $\mathrm{H}$, et al. Impact of hospital consultants poor mental health on patient care. Br J Psychiatry 2007;190:268-9.

13. Maben J, Peccei R, Adams M, et al. Patients' experiences of care and the influence of staff motivation, affect and well-being: Final report NIHR Service Delivery and Organisation programme, 2012. http://www.netscc.ac.uk/hsdr/files/project/SDO FR_08-1819-213 V01.pdf

14. Maben J, Adams M, Peccei R, et al. 'Poppets and parcels': the links between staff experience of work and acutely ill older peoples' experience of hospital care. Int J Older People Nurs 2012;7:83-94.

15. Raleigh VS, Hussey D, Seccombe I, et al. Do associations between staff and inpatient feedback have the potential for improving patient experience? An analysis of surveys in NHS acute trusts in England. Qual Saf Health Care 2009;18:347-54.

16. Australian Government (2018) Healthy workers healthy futures initiative. http://www.healthyworkers.gov.au/ (Last accessed 15 Feb 18).

17. American Nurses Association. 2018 http://www.healthynursehealthyn ation.org (Last accessed 15 Feb 18).

18. Royal College of Physicians (2015)Work and Wellbeing in the NHS: why staff health matters to patient care. 2015. https://www.rcpsych. ac.uk/pdf/RCP-\%20WorkWellbeingNHS.pdf (Last accessed 15 Feb 18).

19. Rao AS, Bhutani G, Dosanjh N, et al, 2016. Psychological Wellbeing and Resilience: Resetting the Balance http://www.bps.org.uk/ system/files/Public\%20files/ar_v10_resetting_the_balance_160516. docx.pdf (Last accessed 15 Feb 2018).

20. Staff Engagement: six building blocks for harnessing the creativity and enthusiasm of NHS staff: The Kings Fund, 2015. https://www. kingsfund.org.uk/sites/default/files/field/field publication file/staffengagement-feb-2015.pdf. (Last accessed 15 Feb 2018).

21. Wales NHS, 2017. Staff psychological wellbeing policy http://www. wales.nhs.uk/sitesplus/documents/862/340-PsychologicalWellbei ngPolicy-ext31.12.17.pdf (Last accessed 15 Feb 18).

22. NICE. Mental wellbeing at work: Public Health Guidance, 2009 https://www.nice.org.uk/guidance/ph22. (Last accessed 15 Feb 18).

23. Robert G, Philippou J, Leamy M, et al. Exploring the adoption of Schwartz Center Rounds as an organisational innovation to improve staff well-being in England, 2009-2015. BMJ Open 2017;7:e014326-10.

24. Lown BA, Manning CF. The schwartz center rounds: evaluation of an interdisciplinary approach to enhancing patient-centered communication, teamwork, and provider support. Acad Med 2010;85:1073-81.

25. Goodrich J. Supporting hospital staff to provide compassionate care: do Schwartz Center Rounds work in English hospitals? J R Soc Med 2012;105:117-22.

26. Maben J, Taylor C, Dawson J, et al, 2018. A realist informed mixed methods evaluation of Schwartz Center Rounds $₫$ in England. First 
Look Summary https://www.journalslibrary.nihr.ac.uk/programmes/ hsdr/130749/\#/ (Last accessed 15 Feb 18).

27. Jones $\mathrm{CE}$, Maben J, Jack RH, et al. A systematic review of barriers to early presentation and diagnosis with breast cancer among black women. BMJ Open 2014;4:e004076.

28. O'Cathain A, Murphy E, Nicholl J. The quality of mixed methods studies in health services research. J Health Serv Res Policy 2008;13:92-8.

29. Arksey H, O'Malley L. Scoping studies: towards a methodological framework. Int J Soc Res Methodol 2005;8:19-32.

30. Ruotsalainen J, Serra C, Marine A, et al. Systematic review of interventions for reducing occupational stress in health care workers. Scand J Work Environ Health 2008;34:169-78.

31. Boorman S. NHS Health and Wellbeing Review. London: Department of Health, 2009.

32. Ruotsalainen JH, Verbeek JH, Mariné $A$, et al. Preventing occupational stress in healthcare workers. Cochrane Database Syst Rev 2015:CD002892.

33. Harvey SB, Laird B, Henderson M, et al. The mental health of health care professionals: A review for the Department of Health. In. London 2009.

34. Anon, 2008. A place to Heal (2008) https://www.nurse.com/blog/ 2008/09/08/a-place-to-heal/ (Last accessed 15 Feb 18).

35. Barnard P. Schwartz Rounds: Librarian Roles and Opportunities. $J$ Hosp Librariansh 2013;13:71-8.

36. Baverstock AC, Finlay FO. Maintaining compassion and preventing compassion fatigue: a practical guide. Arch Dis Child Educ Pract Ed 2016;101:170-4.

37. Berry M, Chaggar R. Introducing schwartz center rounds to watford general hospital - challenging the status quo. Anaesthesia 2014:69:46. Abstract Only.

38. Booth M, 2014. Schwartz Center Rounds - a moment to reflect at St Wilfrid's Hospice eHospice http://www.ehospice.com/uk/Default/ tabid/10697/Articleld/11798 (accessed 15 Feb 2018).

39. Corless IB, Michel TH, Nicholas M, et al. Educating health professions students about the issues involved in communicating effectively: a novel approach. J Nurs Educ 2009;48:367-73.

40. Cornwell J, Goodrich J. Supporting staff to deliver compassionate care using Schwartz Center rounds--a UK pilot. Nurs Times 2010;106:10-12.

41. Davis C. Forum to help staff deal with day-to-day stress. Nurs Manag 2011;18:18-21.

42. Dean E. Time for reflection. Nurs Stand 2013;27:61.

43. Dedier J, Penson R, Williams W, et al. Race, ethnicity, and the patient-caregiver relationship. Oncologist 1999;4:325-31. [included to represent the many publications with the same definition from the Schwartz Center, Massachusetts General Hospital, Boston USA].

44. Deppoliti DI, Côté-Arsenault D, Myers G, et al. Evaluating Schwartz Center Rounds in an urban hospital center. $J$ Health Organ Manag 2015;29:973-87.

45. Foster S. Schwartz Rounds: our experience. Br J Nurs 2015;24:309.

46. Gannon C, Cullen A, Knight A. Contrasting schwartz rounds with clinical ethics: three perspectives on their potential to impact on endof-life care. Nurs Ethics 2014;21:621-3.

47. George MS. Stress in NHS staff triggers defensive inward-focussing and an associated loss of connection with colleagues: this is reversed by Schwartz Rounds. J Compassionate Health Care 2016;3:9.

48. Gibson S. Schwartz center rounds: Focusing on the patient-caregiver relationship. Health Progress. Nov-Dec 2008;2008:40-3.

49. Gishen F, Wood M. Using schwartz center rounds to change hospice culture: does it work? BMJ Supportive \& Palliative Care 2015;1:108.

50. Gishen F, Whitman S, Gill D, et al. Schwartz Centre Rounds: a new initiative in the undergraduate curriculum-what do medical students think? BMC Med Educ 2016;16:246.

51. Goodrich J. Schwartz Center Rounds: Evaluation of the UK Pilots. London: The Kings Fund, 2011. https://www.kingsfund.org.uk/ publications/schwartz-center-rounds-pilot-evaluation. (last accessed 15Ffeb 2018).

52. Goodrich J. Compassionate care and schwartz rounds: the nature of the work-acknowledging it is hard. Nurse Educ Today 2014;34:1185-7.

53. Hargreaves R. "The value of Schwartz Rounds should not be underestimated". Nurs Times 2015;111:11.

54. Johal J. Kindling Kindness for Compassionate Disaster Management. PLOS Currents: Disasters. 2015 http://currents.plos.org/disasters/ article/kindling-kindness-for-compassionate-disaster-management/ (last accessed 15 Feb 2018).
55. Justice S, Rawles J, Brown J, et al. Will introducing 'Schwartz rounds' help foster a culture of compassionate care in the NHS? Nursing Standard. 27(5) 2013;2:26-7.

56. Kaplan K. Powerpoint presentation): Leading important conversations in healthcare: The schwartz center rounds. http://c ymcdn.com/sites/www.odnetwork.org/resource/resmgr/2011_conf_ ppts/g3 - leading important conve.pdf (accessed 15 Feb 2018).

57. Lee KJ, Forbes ML, Lukasiewicz GJ, et al. Promoting Staff Resilience in the Pediatric Intensive Care Unit. Am J Crit Care 2015;24:422-30.

58. Lima J, Cavaliere R, Porensky E. Closing the gap: Bereavement support for families, caregivers and medical staff. Neuro Oncol 2014;16:v182-3.

59. Manning CF, Acker M, Houseman L, et al. Schwartz Center Rounds: Evaluation Report (executive summary. Boston USA: Goodman Research Group Inc, 2008. http://www.theschwartzcenter.org/media/ PTXAAE65CHR5UU4.pdf. (Last accessed 15 Feb 2018).

60. Moore CD, Phillips JM, McCole Phillips J. In these rounds, healthcare professionals heal themselves. J Soc Work End Life Palliat Care 2009;5(3-4):116-25.

61. Mudge B, Stanzler M. Schwartz center rounds and magnet: Enhancing patient- and family-centered care american nurse today, 7(1). 2012 https://www.americannursetoday.com/schwartz-centerrounds-and-magnet-enhancing-patient-and-family-centered-care/ (accessed 1 Mar 2017).

62. Mullick A, Wright A, Watmore-Eve J, et al. Supporting hospice staff: The introduction of schwartz center rounds to a UK hospice setting. European Journal of Palliative Care 2013;20:62-5.

63. Pepper JR, Jaggar SI, Mason MJ, et al. Schwartz Rounds: reviving compassion in modern healthcare. J R Soc Med 2012;105:94-5.

64. Raphael-Grimm, T, Hill, C. Fostering compassionate patient care as an integrated, multidisciplinary outcome: Lessons learned from facilitating "Schwartz Rounds". International Journal of Human Caring 2008;12:97.2-97.

65. Raso R. Improving practice with Schwartz rounds; the DNP degree. Nursing Management 2009;40:56.

66. Reed E, Cullen A, Gannon C, et al. Use of Schwartz Centre Rounds in a UK hospice: Findings from a longitudinal evaluation. $J$ Interprof Care 2015;29:365-6.

67. Rees-Adams D, Hughes L. Introducing schwartz center rounds into a welsh nhs health board: six months in - a reflection on the process and experience to date. Clinical Psychology Forum 2014;263:26-7.

68. Ryan B. Schwartz Center Rounds are one way to boost compassion (Letter). Nursing Standard 2013;27:31.

69. Sanghavi DM. What makes for a compassionate patient-caregiver relationship? Jt Comm J Qual Patient Saf 2006;32:283-92.

70. Shield RR, Tong I, Tomas M, et al. Teaching communication and compassionate care skills: an innovative curriculum for pre-clerkship medical students. Med Teach 2011;33:e408-e416

71. Smyth M. End of shift: goodbye to baby. 2011 https://www.nurse. com/blog/2011/07/11/end-of-shift-goodbye-to-baby-2/ (last accessed 1 Mar 2017)

72. Thompson A. How Schwartz rounds can be used to combat compassion fatigue. Nurs Manag 2013;20:16-20.

73. Whitehead P, Malik R. The impact of schwartz center rounds on moral distress in a large healthcare system (S802). J Pain Symptom Manage 2015;49:461.

74. Wren B. Schwartz Rounds: An intervention with potential to simultaneously improve staff experience and organisational culture. Clinical Psychology Forum 2632014

75. Farr M, Barker R. Can staff be supported to deliver compassionate care through implementing schwartz rounds in community and mental health services? Qual Health Res 2017;27:1652-63.

76. Chadwick RJ, Muncer SJ, Hannon BC, et al. Support for compassionate care: Quantitative and qualitative evaluation of Schwartz Center Rounds in an acute general hospital. JRSM Open 2016;7:205427041664804

77. Babin BJ, Boles JS. The effects of perceived co-worker involvement and supervisor support on service provider role stress, performance and job satisfaction. Journal of Retailing 1996;72:57-75.

78. Wren B. Schwartz Rounds: An intervention with potential to simultaneously improve staff experience and organisational culture. (Special Issue of the British Psychological Society Clinical Psychology Forum on The Francis Report). Clinical Psychology Forum 2014:263.

79. Maben J, Leamy M, Taylor C, et al. Bennett L. (2018) An Organisational guide: Understanding, implementing and sustaining Schwartz Rounds Copyright. King's College London, 2018. 34: https://www.surrey.ac.uk/SchwartzEvaluation. 